Adv. Studies Theor. Phys., Vol. 8, 2014, no. 5, 195 - 213

HIKARI Ltd, www.m-hikari.com

http://dx.doi.org/10.12988/astp.2014.312143

\title{
Composite Fractional Time Evolutions of Cumulants in Fractional Generalized Birth Processes
}

\author{
Hidetoshi Konno and Imre Pázsit ${ }^{1}$ \\ Department of Risk Engineering, Faculty of Information and Systems \\ University of Tsukuba, Tsukuba, Ibaraki 305-8573 Japan \\ Copyright (C) 2014 H. Konno and I. Pázsit. This is an open access article distributed \\ under the Creative Commons Attribution License, which permits unrestricted use, distribu- \\ tion, and reproduction in any medium, provided the original work is properly cited.
}

\begin{abstract}
A fractional generalized pure birth process is studied based on the master equation approach. The exact analytic solution of the generating function of the probability density for the process is given in terms of the Gauss hypergeometric function. The expressions of moments are also obtained in an explicit form. The effects of fixed and distributed initial conditions are also elucidated. The effect of memory is demonstrated quantitatively in terms of the mean, variance, and the Fano factor. It is also shown how to discriminate a fractional generalized birth process from the fractional Yule-Furry process. Further, the appearance of composite fractional time evolutions of cumulants is elucidated in conjunction with the fractional Poisson and the fractional Yule-furry processes.
\end{abstract}

Keywords: fractional generalized birth processes, Master equation, exact solution, composite fractional time evolutions of cumulants

\section{Introduction}

Fractional generalizations of models with long memory in complex systems have been studied extensively [1-11]. There are various approaches for the fractional generalizations:

\footnotetext{
${ }^{1}$ Permanent adress: Chalmers University of Technology, Göteborg, Sweden
} 
(1) A naive method is that the normal time derivative is replaced by a fractional derivative: in this respect (a) fractional Master equation (Saichev and Zaslavsky [5]; Laskin [6], Orsingher and Polito, [7-8], Konno [9]), (b) fractional Fokker-Planck equation (Barkai [10]) and (c) fractional Langevin equation (Franosch et al. [11]) were studied.

(2) When a normal spatial derivative is replaced by a spatial fractional derivative, one obtains a fractional diffusion equation (Lévy diffusion) [3]

(3) When normal spatial and temporal derivatives are replaced by fractional derivatives, one obtains fractional spatial-temporal derivative diffusion equation (Zaslavsky [5]) and various fractional transport equations.

(4) non-stationary (non-homogeneous) generalization of time-functions (Kubo [16], Tokuyama-Mori [17], Konno [12-13], Bedford and Cooke [18])

(5) Conventional Memory function approach based on the Langevin equation (Kubo [14], Mori [15]) and Master equation (Montroll-Schlesinger [3]).

In spite of the numerous studies made, the origin of the fractional derivatives and the theoretical and experimental correspondence have not been clarified completely. This kind of fractional formulation is considered to incorporate the effect of memory in complex systems to understand various unexplained features of complicated dynamics in the real world.

Actually, we have discussed the fractional Poisson process in conjunction with the counting statistics of neural systems [9]. It is found that the Fano factor takes a larger value than 1 (super-Poisson statistics). The waiting time distribution has a fat tail, and the related moments diverge. Then, the logarithmic cumulants are proposed as a convenient measure without divergence [9].

The paper presents the effect of memory upon the nature of fluctuations in a model of fractional generalized birth process (GBP), which is organized as follows. Section 2 describes the model equation, and exact solutions of the generating function for various initial conditions are given. Section 3 gives the expressions of the moments, and the Fano factor associated with various initial condition is given. Section 4 discusses the appearance of composite fractional time evolution of cumulants, the method of discriminating the fractional GBP from the fractional Yule-Furry process and the fractional Poisson process in detail. Section 5 is devoted to summary and remarks.

\section{Fractional Generalized Linear Birth Process}

\section{1 $\quad$ Model}

Let us consider the fractional generalized birth process (FGBP) with fractional derivative $(0<\mu \leq 1)$

$$
{ }_{0} D_{t}^{\mu} p_{\mu}(n, t)=[\alpha(n-1)+\beta] p_{\mu}(n-1, t)-(\alpha n+\beta) p_{\mu}(n, t), \quad(n \geq 1)
$$


and ${ }_{0} D_{t}^{\mu} p_{\mu}(0, t)=-\beta p_{\mu}(0, t),(n=0)$, where ${ }_{0} D_{t}^{\mu}$ is the Caputo fractional derivative defined by

$$
{ }_{0} D_{t}^{\mu} f(t) \equiv \frac{1}{\Gamma(1-\mu)} \int_{0}^{t} \frac{f^{\prime}(\tau)}{(t-\tau)^{\mu}} d \tau
$$

The other definition of fractional derivative due to Riemann-Liouville is defined by

$$
{ }_{0} \mathcal{D}_{t}^{\mu} f(t) \equiv \frac{1}{\Gamma(1-\mu)} \frac{d}{d t} \int_{0}^{t} \frac{f(\tau)}{(t-\tau)^{\mu}} d \tau .
$$

The relation between the two derivatives is

$$
{ }_{0} \mathcal{D}_{t}^{\mu} f(t)={ }_{0} D_{t}^{\mu} f(t)+\sum_{k=0}^{m-1} \frac{t^{k-\mu}}{\Gamma(k-\mu+1)} f^{(k)}(0+) .
$$

Rewriting Eq.(1) in the form,

$$
\frac{\partial}{\partial t} p_{\mu}(n, t)={ }_{0} D_{t}^{1-\mu}\left[(\alpha(n-1)+\beta) p_{\mu}(n-1, t)-(\alpha n+\beta) p_{\mu}(n, t)\right],
$$

one can see that the model in Eq.(5), a fractional generalized birth process, can be regarded as a memory function type Master equation.

Since Caputo's definition gives

$$
{ }_{0} D_{t}^{\mu} 1 \equiv 0
$$

it is quite natural to consider the physical processes depending on the initial value. When the Riemann-Liouville fractional derivative is adopted, one must insert an additional term in the right hand side of the model in Eq.(1) on account of the relation:

$$
{ }_{0} \mathcal{D}_{t}^{\mu} 1=\frac{t^{-\mu}}{\Gamma(1-\mu)} .
$$

In considering the initial transient under an environment with long-memory, it is important to set up the initial condition (IC) properly. Here, we will consider two simple cases: case 1, i.e.,

$$
p_{\mu}(n, 0)=\delta_{n, n_{0}}
$$

and case 2 , i.e.,

$$
p_{\mu}(n, 0)=\delta_{n, n_{0}} \frac{\theta^{n}}{n !} e^{-\theta} .
$$

We will refer to case 1 as either IC0 $\left(n_{0}=0\right)$ or IC1 $\left(n_{0} \neq 0\right)$, and to case 2 as IC2. When the initial condition is controllable in experiments, one can choose case 1 . On the other hand, when the initial condition is not controllable in experiments, one must choose, for example, case 2. Here we will not consider more sophisticated initial distributions for brevity. The fractional linear birth process in Eq.(1) with $\beta=0$ under the initial condition $n_{0}=1$ is studied by Orsingher and Polito [7] from the mathematical point of view. 


\subsection{Inverse Lévy Transform}

An inverse Lévy transform is defined $[5,10]$ by

$$
p_{\mu}(n, t)=\int_{0}^{\infty} K_{\mu}(\tau, t) p_{1}(n, \tau) d \tau,
$$

where the integral kernel $K_{\mu}(\tau, t)$ is expressed in terms of the one-sided cumulative Lévy distribution $L_{\mu}(z)$ (cf. Appendix A):

$$
K_{\mu}(\tau, t)=\frac{d}{d \tau}\left[1-L_{\mu}\left(t / \tau^{1 / \mu}\right)\right] .
$$

When the analytic solution for $\mu=1$ (i.e., $\left.p_{1}(n, t)\right)$ is given, the solution of the FGBP $p_{\mu}(n, t)$ can be obtained. The generating function in the FGBP (1) can be derived from $g_{1}(z, t)$ as

$$
g_{\mu}(z, t)=\int_{0}^{\infty} K_{\mu}(\tau, t) g_{1}(z, \tau) d \tau
$$

\subsection{Probability mass}

Now let us derive the exact solution for the probability mass function (pmf) $p_{\mu}(n, t)$ via the generating function $g_{1}(z, t)$ for $\mu=1$, which is subjected to

$$
\frac{\partial}{\partial t} g_{1}(z, t)=\alpha z(z-1) \frac{\partial}{\partial z} g_{1}(z, t)+\beta(z-1) g_{1}(z, t)
$$

The solution under $\operatorname{IC} 1\left(p_{1}(n, 0)=\delta_{n, n_{0}}\right)$ is given by

$$
g_{1}(z, t)=z^{n_{0}}(\exp (-\alpha t))^{n_{0}+\frac{\beta}{\alpha}}(1-z(1-\exp (-\alpha t)))^{-\left(n_{0}+\frac{\beta}{\alpha}\right)}
$$

With the use of the inverse Lévy transform of Eq.(10), one obtains $g_{\mu}[z, s]$ in the Laplase domain for the IC1 (i.e., $g_{\mu}(z, 0)=z^{n_{0}}$ ) as

$$
\begin{gathered}
g_{\mu}[z, s]=z^{n_{0}}\left(\frac{s^{\mu-1}}{\alpha}\right) \int_{0}^{1}(1-x)^{\frac{s^{\nu}}{\alpha}+n_{0}+\frac{\beta}{\alpha}-1}(1-x z)^{-\left(n_{0}+\frac{\beta}{\alpha}\right)} d x, \\
=z^{n_{0}}\left(\frac{s^{\mu-1}}{s^{\mu}+n_{0} \alpha+\beta}\right) F\left(n_{0}+\frac{\alpha}{\beta}, 1, \frac{s^{\mu}}{\alpha}+n_{0}+\frac{\beta}{\alpha}+1 ; z\right)
\end{gathered}
$$

where $F(a, b, c ; z)$ is Euler's integral representation of the Gauss hypergeometric function defined by

$$
F(a, b, c ; z)=\frac{\Gamma(c)}{\Gamma(b) \Gamma(c-b)} \int_{0}^{1} x^{b-1}(1-x)^{c-b-1}(1-z x)^{-a} d x
$$


where $\Gamma(z)$ is the Gamma function: $\Gamma(z)=\int_{0}^{\infty} t^{z-1} e^{-t} d t$. The probability mass $p_{\mu}[z, s]$ under IC1 $\left(p_{\mu}(n, 0)=\delta_{n, n_{0}}\right)$ is obtained from $g_{\mu}[z, s]$ as

$$
p_{\mu}[n, s]=\left(\begin{array}{c}
n+\frac{\beta}{\alpha}-1 \\
n-n_{0}
\end{array}\right) \cdot \frac{s^{\mu-1}}{\alpha} \cdot B\left(n-n_{0}+1, \frac{s^{\mu}}{\alpha}+n_{0}+\frac{\beta}{\alpha}\right),
$$

where $B(x, y)$ is the Beta function defined by

$$
B(x, y)=\int_{0}^{1} u^{x-1}(1-u)^{y-1} d u .
$$

The explicit expression of the pmf in the time domain can be obtained from this in terms of the Mittag-Leffler function.

When the initial condition is not controllable, one must take average over the initial values. For the initial condition $(\mathrm{IC} 2) p(n, 0)=\delta_{n, n_{0}}(\theta)^{n} \exp (-\theta) / n$ ! (i.e., a Poisson distribution with the parameter $\theta$ ), one obtains the generating function which is averaged over the initial condition as

$$
\overline{g_{1}(z, t)}=[\exp (-\alpha t)]^{\frac{\beta}{\alpha}}(1-z[1-\exp (-\alpha t)])^{-\frac{\beta}{\alpha}} \exp \left(-\frac{(1-z) \theta}{1-z[1-\exp (-\alpha t)]}\right) .
$$

By virtue of the Levy transform, we have

$$
\overline{g_{\mu}[z, s]}=\frac{s^{\mu-1}}{\alpha} \int_{0}^{1}(1-x)^{\frac{s^{\mu}}{\alpha}+\frac{\beta}{\alpha}-1}(1-x z)^{-\frac{\beta}{\alpha}} \exp \left(-\frac{1-z}{1-x z} \theta\right) d x .
$$

This solution can be written as (cf. Appendix B for derivation)

$$
\overline{g_{\mu}[z, s]}=\frac{s^{\mu-1}}{s^{\mu}+\alpha} F_{g}\left(\frac{\alpha}{\beta}, 1, \frac{s^{\mu}}{\alpha}+\frac{\beta}{\alpha}+1, \theta ; z\right),
$$

in terms of a generalized hypergeometric function defined by

$$
F_{g}(a, b, c, \theta ; z)=\frac{\Gamma(c)}{\Gamma(b) \Gamma(c-b)} \int_{0}^{1} x^{b-1}(1-x)^{c-b-1}(1-z x)^{-a} e^{-\frac{1-z}{1-x z} \theta} d x .
$$

An explicit expression for $\overline{p_{\mu}[n, s]}$ can be obtained by the formula,

$$
\overline{p_{\mu}[n, s]}=\left.\frac{1}{n !} \frac{\partial^{n}}{\partial z^{n}} \overline{g_{\mu}[z, s]}\right|_{z=0}
$$

The general expression becomes complicated. An explicit expression for $\overline{p_{\mu}(n, t)}$ can be obtained recursively $(n=0,1,2, \ldots)$ in terms of the Migttag-Leffler function, e.g.,

$$
\overline{p_{\mu}(1, t)}=\theta e^{-\theta} E_{\mu}\left(-(\alpha+\beta) t^{\mu}\right)+\frac{\beta}{\alpha} e^{-\theta}\left(E_{\mu}\left(-\beta t^{\mu}\right)-E_{\mu}\left(-(\alpha+\beta) t^{\mu}\right)\right) .
$$




\section{Moments and Fano Factor}

\subsection{Moments}

The moments of the counting number during the time interval $T$ in the FGBP under IC0 $\left(n_{0}=0\right)$ are obtained with the generating function in Eq.(15) as

$$
\begin{gathered}
\langle n(T)\rangle=\left(\frac{\beta}{\alpha}\right)\left(E_{\mu}\left(\alpha T^{\mu}\right)-1\right) \\
\left\langle n(T)^{2}\right\rangle=\left(\frac{\beta}{\alpha}\right)\left(E_{\mu}\left(2 \alpha T^{\mu}\right)-E_{\mu}\left(\alpha T^{\mu}\right)\right)+\left(\frac{\beta}{\alpha}\right)^{2}\left(E_{\mu}\left(2 \alpha T^{\mu}\right)-2 E_{\mu}\left(\alpha T^{\mu}\right)+1\right),
\end{gathered}
$$

and

$$
\sigma_{n}^{2}(T)=\left(\frac{\beta}{\alpha}\right)\left(E_{\mu}\left(2 \alpha T^{\mu}\right)-E_{\mu}\left(\alpha T^{\mu}\right)\right)+\left(\frac{\beta}{\alpha}\right)^{2}\left(E_{\mu}\left(2 \alpha T^{\mu}\right)-E_{\mu}\left(\alpha T^{\mu}\right)^{2}\right)
$$

where $\langle X(n)\rangle$ denotes the statistical average of a variable $X(n)$ as a function of $n:\langle X(n)\rangle=\sum_{n=0}^{\infty} X(n) P(n, t)$. It is an interesting observation that the second moment in Eq. (27), hence also the variance, is composed of two terms: One is proportional to $(\beta / \alpha)$, and the other is to $(\beta / \alpha)^{2}$. When $\mu \rightarrow 1$, the last term with $(\beta / \alpha)^{2}$ in Eq.(28) tend to vanish.

It is easy to see that the variance becomes for $\mu=1$ as

$$
\langle n(T)\rangle=\frac{\beta}{\alpha}\left(e^{\alpha T}-1\right)
$$

and

$$
\sigma_{n}^{2}(T)=\frac{\beta}{\alpha}\left(e^{\alpha T}-1\right) e^{\alpha T}
$$

The moments of the counting number during the interval $T$ in the FGBP under IC1 $\left(n_{0} \neq 0\right)$ are also obtained by the generating function as

$$
\begin{gathered}
\langle n(T)\rangle=n_{0} E_{\mu}\left(\alpha T^{\mu}\right)+\left(\frac{\beta}{\alpha}\right)\left(E_{\mu}\left(\alpha T^{\mu}\right)-1\right) \\
\left\langle n(T)^{2}\right\rangle=\left(n_{0}+\frac{\beta}{\alpha}\right)\left(E_{\mu}\left(2 \alpha T^{\mu}\right)-E_{\mu}\left(\alpha T^{\mu}\right)\right)+\left(n_{0}+\frac{\beta}{\alpha}\right)^{2}\left(E_{\mu}\left(2 \alpha T^{\mu}\right)-2 E_{\mu}\left(\alpha T^{\mu}\right)+1\right) \\
+2 n_{0}\left(n_{0}+\frac{\beta}{\alpha}\right)\left(E_{\mu}\left(\alpha T^{\mu}\right)-1\right)+n_{0}^{2} .
\end{gathered}
$$

where $\langle X(n)\rangle$ is defined as above. It is easy to see that the variance depends on $n_{0}$ as

$\sigma_{n}^{2}(T)=\left(n_{0}+\frac{\beta}{\alpha}\right)\left(E_{\mu}\left(2 \alpha T^{\mu}\right)-E_{\mu}\left(\alpha T^{\mu}\right)\right)+\left(n_{0}+\frac{\beta}{\alpha}\right)^{2}\left(E_{\mu}\left(2 \alpha T^{\mu}\right)-E_{\mu}\left(\alpha T^{\mu}\right)^{2}\right)$. 

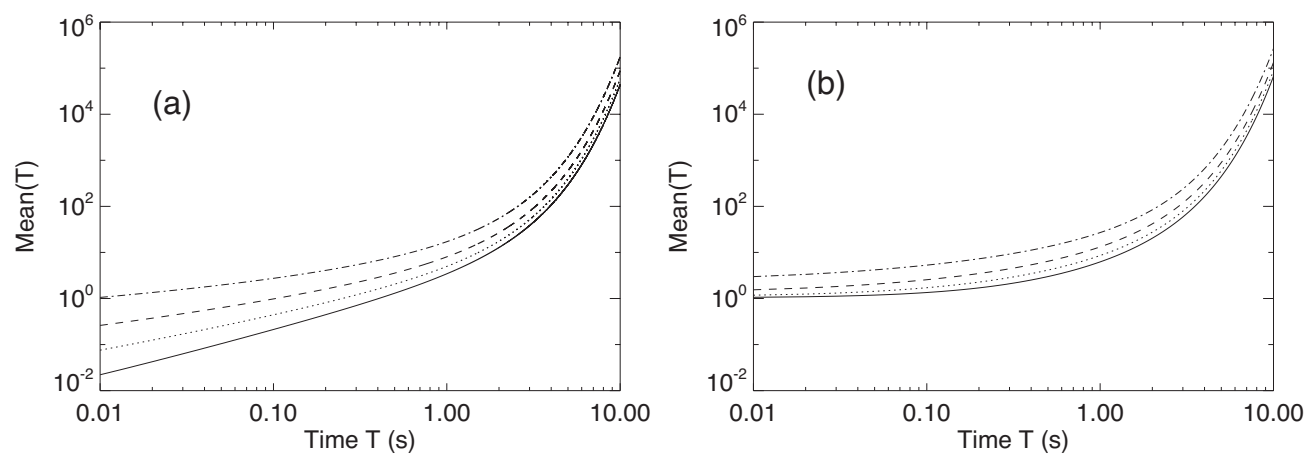

Figure 1: The variation of the mean as a function of $T$ for (a) $n_{0}=0$ and (b) $n_{0}=1$ in Eq.(31) with $\alpha=1$ and $\beta=2$. Solid line $\mu=1$, dotted line $\mu=0.75$, dashed line $\mu=0.50$ and dash-dot line $\mu=0.25$.
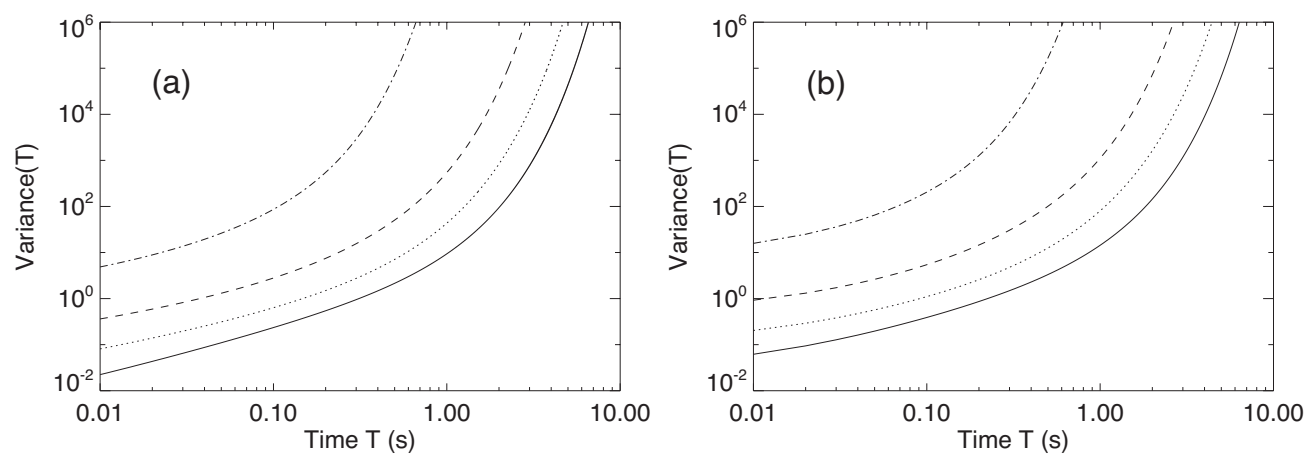

Figure 2: The variation of the variance as a function of $T$ for (a) $n_{0}=0$ and (b) $n_{0}=1$ in Eq.(33) with $\alpha=1$ and $\beta=2$. Solid line $\mu=1$, dotted line $\mu=0.75$, dashed line $\mu=0.50$ and dash-dot line $\mu=0.25$.

Figure 1 shows the variation of the mean $\langle n(T)\rangle$ as a function of $T$ for (a) $n_{0}=0$ and (b) $n_{0}=1$ in Eq.(31) with $\alpha=1$ and $\beta=2$. Solid line $\mu=1$, dotted line $\mu=0.75$, dashed line $\mu=0.50$ and dash-dot line $\mu=0.25$. It is clear that the growth rate increases as the value of $\mu$ decreases.

Figure 2 shows the variation of the variance as a function of $T$ for (a) $n_{0}=0$ and (b) $n_{0}=1$ in Eq.(33) with $\alpha=1$ and $\beta=2$. Solid line $\mu=1$, dotted line $\mu=0.75$, dashed line $\mu=0.50$ and dash-dot line $\mu=0.25$. It is clear that the growth rate increases as the value of $\mu$ decreases. The profiles of the two types of evolution are slightly different due to the $n_{0}$-dependence.

Figure 3 shows the features of the Mittag-Leffler function (a) $E_{\mu}\left(-T^{\mu}\right)$ and (b) $E_{\mu}\left(T^{\mu}\right)$ as a function of time $T$. Solid line $\mu=1$, dotted line $\mu=0.75$, dashed line $\mu=0.50$ and dash-dot line $\mu=0.25$. One can see how the features of decreasing and increasing vary as a function of value of the parameter $\mu$.

When IC2 is adopted, one obtains the result with the generating function 

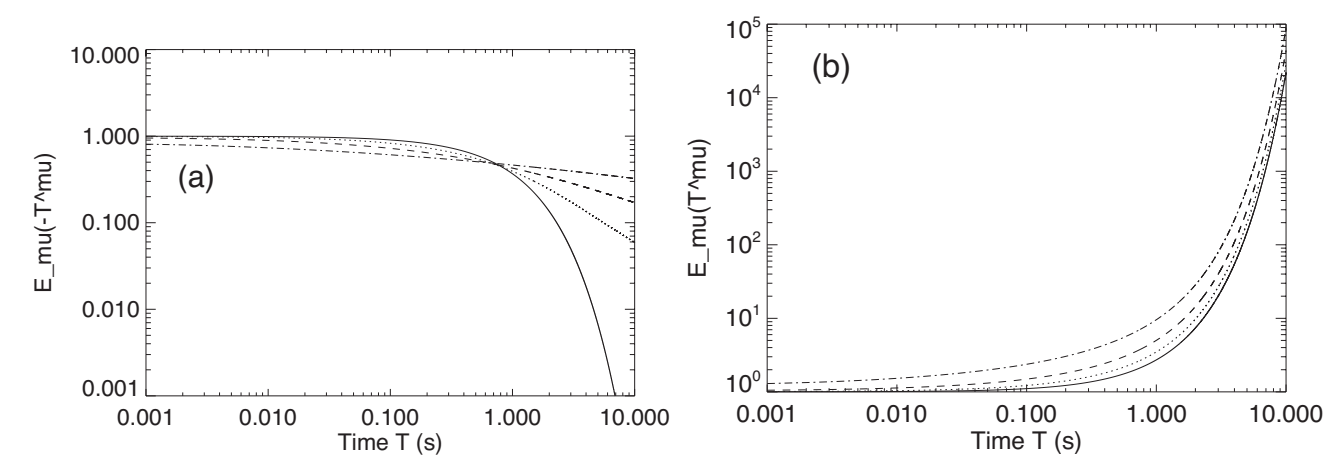

Figure 3: The feature of the Mittag-Leffler function (a) $E_{\mu}\left(-T^{\mu}\right)$ and (b) $E_{\mu}\left(T^{\mu}\right)$ as a function of time $T$. Solid line $\mu=1$, dotted line $\mu=0.75$, dashed line $\mu=0.50$ and dash-dot line $\mu=0.25$.

in Eq.(21) as

$$
\begin{gathered}
\overline{\langle n(T)\rangle}=\theta E_{\mu}\left(\alpha T^{\mu}\right)+\left(\frac{\beta}{\alpha}\right)\left(E_{\mu}\left(\alpha T^{\mu}\right)-1\right) \\
\overline{\left\langle n(T)^{2}\right\rangle}=\left(\theta+\frac{\beta}{\alpha}\right)\left(E_{\mu}\left(2 \alpha T^{\mu}\right)-E_{\mu}\left(\alpha T^{\mu}\right)\right)+\left(\theta+\frac{\beta}{\alpha}\right)^{2}\left(E_{\mu}\left(2 \alpha T^{\mu}\right)-2 E_{\mu}\left(\alpha T^{\mu}\right)+1\right) \\
+2 \theta\left(\theta+\frac{\beta}{\alpha}\right)\left(E_{\mu}\left(\alpha T^{\mu}\right)-1\right)+\theta^{2}+\theta E_{\mu}\left(2 \alpha T^{\mu}\right)
\end{gathered}
$$

and

$$
\begin{gathered}
\overline{\sigma_{n}^{2}(T)}=\left(\theta+\frac{\beta}{\alpha}\right)\left(E_{\mu}\left(2 \alpha T^{\mu}\right)-\right. \\
\left.E_{\mu}\left(\alpha T^{\mu}\right)\right)+\left(\theta+\frac{\beta}{\alpha}\right)^{2}\left(E_{\mu}\left(2 \alpha T^{\mu}\right)-E_{\mu}\left(\alpha T^{\mu}\right)^{2}\right) \\
+\theta E_{\mu}\left(2 \alpha T^{\mu}\right) .
\end{gathered}
$$

The averaged variance $\overline{\sigma_{n}^{2}(T)}$ in Eq.(36) does not take the same form as the variance $\sigma_{n}^{2}(T)$ in Eq.(33) due to the last term of Eq.(36). This term comes from the averaging effect over the distribution of initial value $n_{0}$ (cf. Appendix $\mathrm{C}$ for derivation). When the distribution of initial value is taken into account (IC2), more additional terms appear in the expressions of higher order moments (See section 4.3).

\subsection{Fano factor}

The nature of fluctuations is often discussed in conjunction with the Fano factor [17-22]. Let the sequence of event numbers (counts) be denoted by $\left\{Z_{n}\right\}$. The Fano factor $F F(T)$ in the time interval $T$ is defined as the eventnumber variance divided by the event-number mean:

$$
F F(T) \equiv \frac{\operatorname{Var}\left(Z_{n}(T)\right)}{E\left[Z_{n}(T)\right]}=\frac{\sigma_{\mu}^{2}(T)}{\langle n(T)\rangle} .
$$


The quantity $F F(T)$ has been used as an indicator of random, coherent and squeezed states (i.e., super-Poisson $F F(T)>1$, Poisson $F F(T)=1$ and subPoisson $F F(T)<1$ statistics) of light in the photon counting processes.

When $p(n, 0)=\delta_{n, 0}$ (i.e., the IC0 with $n_{0}=0$ ), one obtains

$$
F F(T)=\frac{E_{\mu}\left(2 \alpha T^{\mu}\right)-E_{\mu}\left(\alpha T^{\mu}\right)}{E_{\mu}\left(\alpha T^{\mu}\right)-1}+\left(\frac{\beta}{\alpha}\right) \frac{E_{\mu}\left(2 \alpha T^{\mu}\right)-E_{\mu}\left(\alpha T^{\mu}\right)^{2}}{E_{\mu}\left(\alpha T^{\mu}\right)-1} .
$$

Namely, the FGBP process $(0<\mu<1)$ in the initial condition $p(n, 0)=\delta_{n, 0}$ is subject to the super-Poissonian statistics $(F F(T)>1)$. One must recall that when $\mu=1$, i.e., in the FGBPprocess, $\langle n(T)\rangle=\sigma_{n}^{2}(T)=F F(T)=\exp (\alpha T)$.

When using IC1 with the initial value $n_{0}=1\left(p(n, 0)=\delta_{n, 1}\right)$, one obtains

$$
\begin{gathered}
F F(T)=\left(1+\frac{\beta}{\alpha}\right) \frac{E_{\mu}\left(2 \alpha T^{\mu}\right)-E_{\mu}\left(\alpha T^{\mu}\right)}{E_{\mu}\left(\alpha T^{\mu}\right)+\left(1+\frac{\beta}{\alpha}\right)\left(E_{\mu}\left(\alpha T^{\mu}\right)-1\right)} \\
+\left(1+\frac{\beta}{\alpha}\right)^{2} \frac{E_{\mu}\left(2 \alpha T^{\mu}\right)-E_{\mu}\left(\alpha T^{\mu}\right)^{2}}{E_{\mu}\left(\alpha T^{\mu}\right)+\left(1+\frac{\beta}{\alpha}\right)\left(E_{\mu}\left(\alpha T^{\mu}\right)-1\right)} .
\end{gathered}
$$

Namely, the FGBP process $(0<\mu<1)$ in the initial condition $p(n, 0)=\delta_{n, n_{0}}$ is subject to the $F F(T)$ described above. The first term is independent of the initial value $n_{0}$. On the other hand, the second term depends on $n_{0}$. This is an interesting observation in this system with memory in Eq.(1). One must recall that when $\mu=1$, i.e., in the FGBP process for $n_{0}=0$ gives $F F(T)=\exp (\alpha T)$ and for $n_{0}=1$ gives $F F(T)=(1+\beta / \alpha) e^{\alpha T}\left(e^{\alpha T}-1\right) /\left(e^{\alpha T}+(\beta / \alpha)\left(e^{\alpha T}-1\right)\right)$.

Figure 4 shows the variation of the Fano factor as a function of $T$ for (a) $n_{0}=0$ in Eq.(38) and (b) $n_{0}=1$ in Eq.(39) with $\alpha=1$ and $\beta=2$. Solid line $\mu=1$, dotted line $\mu=0.75$, dashed line $\mu=0.50$ and dash-dot line $\mu=0.25$. It is clear that the growth rate increases as the value of the parameter $\mu$ decreases.

\section{Discussion}

\subsection{Comparison with fractional Poisson process}

As is shown in the previous papers, the mean and the variance of the fractional Poisson process $(\mathrm{FPP})$ under IC1 $\left(p_{\mu}(n, 0)=\delta_{n, n_{0}}\right)$ becomes $[9]$

$$
\langle n(T)\rangle=n_{0}+\frac{\beta T^{\mu}}{\Gamma(\mu+1)}
$$

and

$$
\sigma_{n}^{2}(T)=\frac{\beta T^{\mu}}{\Gamma(\mu+1)}+\beta^{2}\left(\frac{2}{\Gamma(2 \mu+1)}-\frac{1}{\Gamma(\mu+1)^{2}}\right) T^{2 \mu}
$$



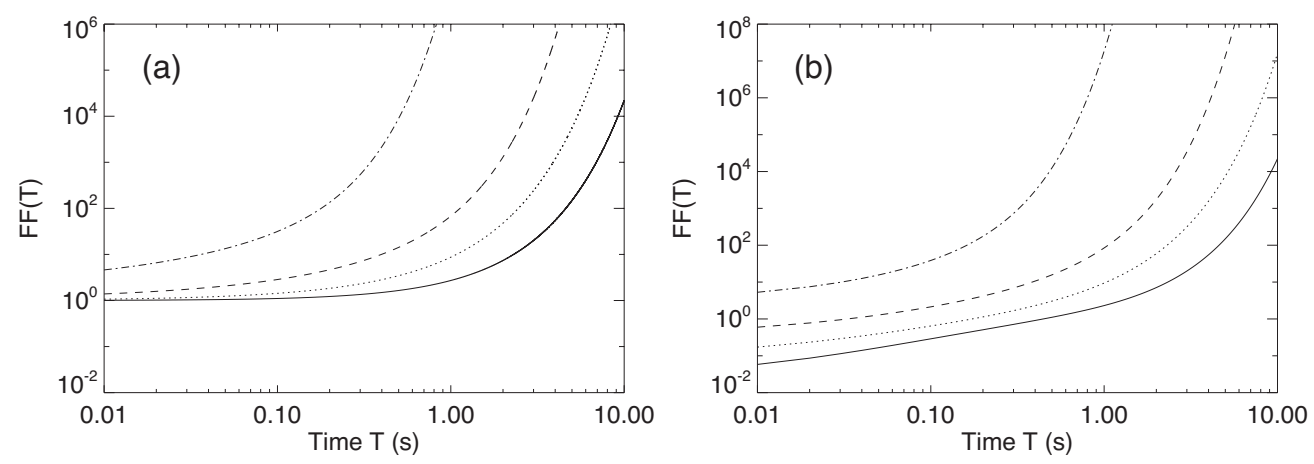

Figure 4: The variation of the Fano factor as a function of $T$ for (a) $n_{0}=0$ in Eq.(38) and (b) $n_{0}=1$ in Eq.(39) with $\alpha=1$ and $\beta=2$. Solid line $\mu=1$, dotted line $\mu=0.75$, dashed line $\mu=0.50$ and dash-dot line $\mu=0.25$.

The variance is independent of $n_{0}$. The nonlinear mode of evolution appears in the right hand side of Eq.(41). This term vanishes when $\mu=1$. This is the origin of the super-Poisson nature $(F F(T)>1)$ in the FPP.

It is an interesting observation that the third order cumulant [19] becomes

$$
\begin{aligned}
C_{3}(T) \equiv & \left\langle(n(T)-\langle n(T)\rangle)^{3}\right\rangle=\frac{\beta T^{\mu}}{\Gamma(\mu+1)}+3 \beta^{2}\left(\frac{2}{\Gamma(2 \mu+1)}-\frac{1}{\Gamma(\mu+1)^{2}}\right) T^{2 \mu} \\
& +2 \beta^{3}\left(\frac{3}{\Gamma(1+3 \mu)}-\frac{3}{\Gamma(2 \mu+1) \Gamma(1+\mu)}+\frac{1}{\Gamma(\mu+1)^{3}}\right) T^{3 \mu}
\end{aligned}
$$

The cumulant $C_{3}(T)$ is also independent of $n_{0}$. The second and the third term can be regarded as the nonlinear evolution modes in the FPP which vanish in the limit $\mu \rightarrow 1$.

Figure 5 shows the variation of the mean in the cases of (a) $n_{0}=0$ and (b) $n_{0}=1$ in Eq.(40) as a function of $T$ with $\beta=1$ for $\mu=1.0,0.75,0.50$ and 0.25 . One can see the simple scaling law in the Fano factor for large values of $T$ in the FPP.

Figure 6 shows the variation of the variance in the cases of (a) $n_{0}=0$ and (b) $n_{0}=1$ in Eq.(41) as a function of $T$ with $\beta=1$ for $\mu=1.0,0.75,0.50$ and 0.25. The existence of two nonlinear modes of evolution is evident from the two different scaling laws in the FPP. Only one picture is shown since there is no $n_{0}$-dependence in the expression of the variance.

Figure 7 shows the variation of the Fano factor in the cases of (a) $n_{0}=0$ and (b) $n_{0}=1$ as a function of $T$ with $\beta=1$ for $\mu=1.0,0.75,0.50$ and 0.25 . It is clear from the figure that the origin of the super-Poisson nature in the fractional Poisson process can be ascribed to the appearance of the nonlinear mode of evolution. 

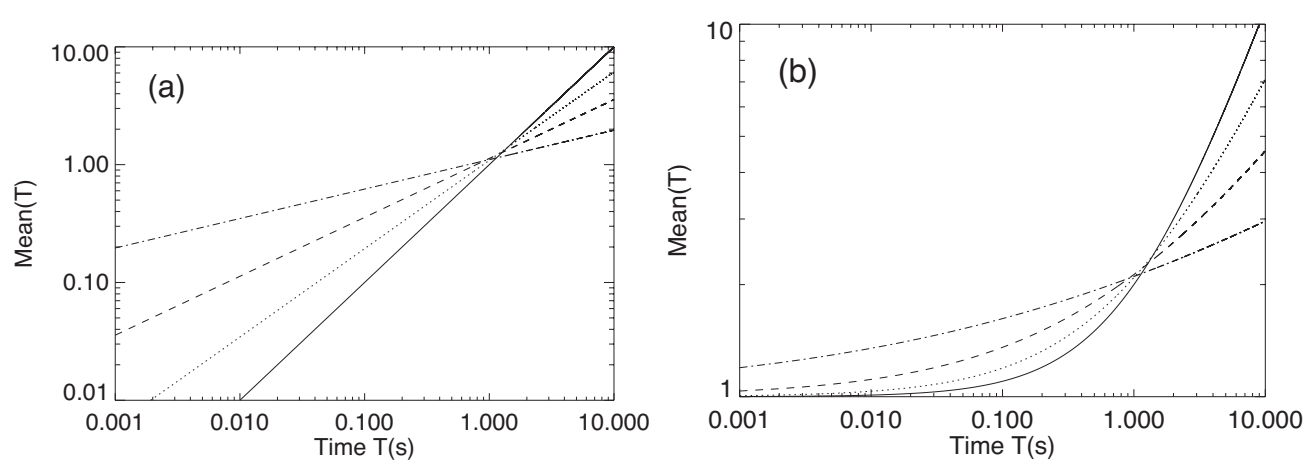

Figure 5: The variation of the mean in the cases of (a) $n_{0}=0$ and (b) $n_{0}=1$ in Eq.(40) as a function of $T$ with $\beta=1$ for $\mu=1.0,0.75,0.50$ and 0.25 .

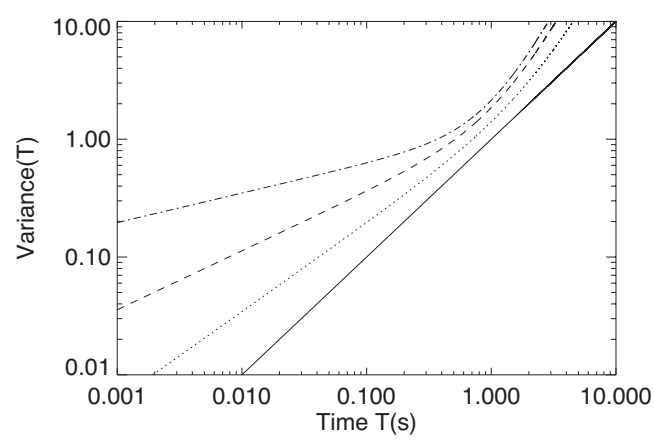

Figure 6: The variation of the variance in the cases of $n_{0}=0$ and $n_{0}=1$ in Eq.(41) as a function of $T$ with $\beta=1$ for $\mu=1.0,0.75,0.50$ and 0.25 .
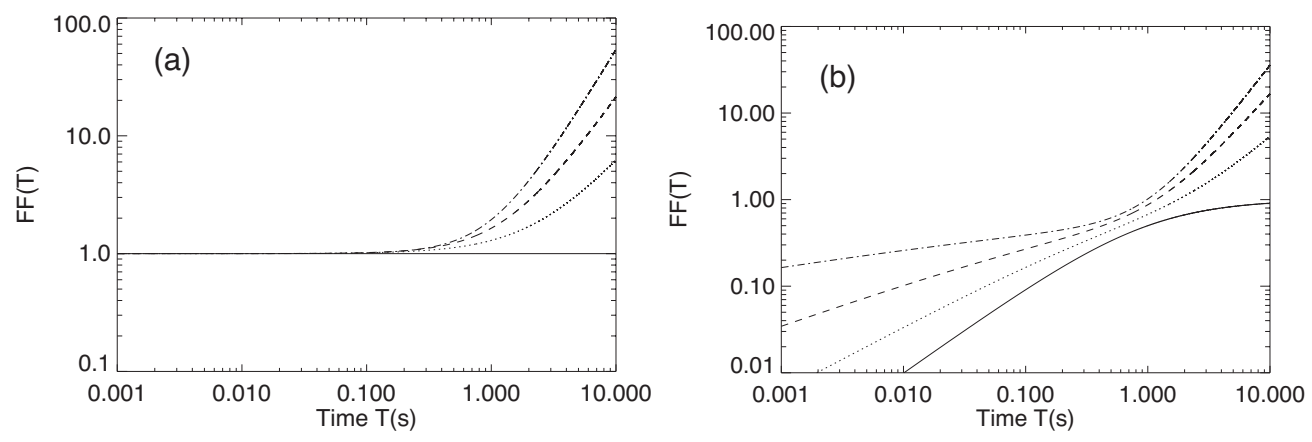

Figure 7: The variation of the Fano factor in the cases of (a) $n_{0}=0$ and (b) $n_{0}=1$ as a function of $T$ with $\beta=1$ for $\mu=1.0,0.75,0.50$ and 0.25 . 


\subsection{Comparison with fractional Yule-Furry process}

Compare now these results with the situation in the case of fractional YuleFurry process (FYFP). The FYFP under the initial condition $p_{\mu}(n, 0)=\delta_{n, 1}$ $\left(n_{0}=1\right)$ has been studied by Orsingher and Polito [7]. The mean and the variance under the initial condition are given by

$$
\langle n(T)\rangle=E_{\mu}\left(\alpha T^{\mu}\right)
$$

and

$$
\sigma_{n}^{2}(T)=2 E_{\mu}\left(2 \alpha T^{\mu}\right)-E_{\mu}\left(\alpha T^{\mu}\right)-E_{\mu}\left(\alpha T^{\mu}\right)^{2} .
$$

Rewriting the expression of the variance in the form,

$$
\sigma_{n}^{2}(T)=\left(E_{\mu}\left(2 \alpha T^{\mu}\right)-E_{\mu}\left(\alpha T^{\mu}\right)\right)+\left(E_{\mu}\left(2 \alpha T^{\mu}\right)-E_{\mu}\left(\alpha T^{\mu}\right)^{2}\right),
$$

one can see that the last term tends to vanish as $\mu \rightarrow 1$. One can expect that $\left(E_{\mu}\left(2 \alpha T^{\mu}\right)-E_{\mu}\left(\alpha T^{\mu}\right)^{2}\right)$ is the nonlinear mode of evolution.

\subsection{Composite fractional time evolutions in FGBP}

The result of the fractional generalized birth process (FGBP) in Section 3.1 shows that the appearance of the term with the nonlinear coefficient $(\beta / \alpha)^{2}$ is due to the memory effect under the interaction of two transition terms with $\alpha$ and $\beta$. Putting $n_{0}=1$ in Eqs.(31) and (33), we have

$$
\langle n(T)\rangle=1+\left(1+\frac{\beta}{\alpha}\right)\left(E_{\mu}\left(\alpha T^{\mu}\right)-1\right)
$$

and

$\sigma_{n}^{2}(T)=\left(1+\frac{\beta}{\alpha}\right)\left(E_{\mu}\left(2 \alpha T^{\mu}\right)-E_{\mu}\left(\alpha T^{\mu}\right)\right)+\left(1+\frac{\beta}{\alpha}\right)^{2}\left(E_{\mu}\left(2 \alpha T^{\mu}\right)-E_{\mu}\left(\alpha T^{\mu}\right)^{2}\right)$.

In summarizing the results, the evolution function of the mean becomes

$$
M_{1}(1)=\left(E_{\mu}\left(\alpha T^{\mu}\right)-1\right)
$$

In the case of variance, there are two different nonlinear modes of evolution:

$$
M_{2}(1)=\left(E_{\mu}\left(2 \alpha T^{\mu}\right)-E_{\mu}\left(\alpha T^{\mu}\right)\right)
$$

and

$$
M_{2}(2)=\left(E_{\mu}\left(2 \alpha T^{\mu}\right)-E_{\mu}\left(\alpha T^{\mu}\right)^{2}\right) .
$$


One can see that $M_{2}(1) \rightarrow e^{2 \alpha T}-e^{\alpha T}$ and $M_{2}(2) \rightarrow 0$ as $\mu \rightarrow 1$. The result suggests that there are three different nonlinear modes of evolution appearing in the third order cumulant. Actually, the expression of the third order cumulant [19] $C_{3}(T)$ for IC1 with $n_{0}=1$ is calculated in the form:

$$
C_{3}(T)=\left(1+\frac{\beta}{\alpha}\right) M_{3}(1)+\left(1+\frac{\beta}{\alpha}\right)^{2} M_{3}(2)+\left(1+\frac{\beta}{\alpha}\right)^{3} M_{3}(3)
$$

where

$$
\begin{gathered}
M_{3}(1)=\left(2 E_{\mu}\left(3 \alpha T^{\mu}\right)-3 E_{\mu}\left(2 \alpha T^{\mu}\right)+E_{\mu}\left(\alpha T^{\mu}\right)\right), \\
M_{3}(2)=3\left(E_{\mu}\left(3 \alpha T^{\mu}\right)-E_{\mu}\left(2 \alpha T^{\mu}\right) E_{\mu}\left(\alpha T^{\mu}\right)\right)-3 M_{2}(2)
\end{gathered}
$$

and

$$
M_{3}(3)=\left(E_{\mu}\left(3 \alpha T^{\mu}\right)+2 E_{\mu}\left(\alpha T^{\mu}\right)^{3}-3 E_{\mu}\left(2 \alpha T^{\mu}\right) E_{\mu}\left(\alpha T^{\mu}\right)\right) .
$$

These are the three nonlinear modes of evolution in the FGBP. One can also see that $M_{3}(1) \rightarrow e^{\alpha T}\left(2 e^{\alpha T}-1\right)\left(e^{\alpha T}-1\right), M_{3}(2) \rightarrow 0$ and $M_{3}(3) \rightarrow 0$ as $\mu \rightarrow 1$. The result for the fourth order cumulant can be inferred from these.

The expression of the third order cumulant for IC2 is obtained in the form:

$$
\begin{aligned}
\overline{C_{3}(T)}=\left(\theta+\frac{\beta}{\alpha}\right) M_{3}(1)+\left(\theta+\frac{\beta}{\alpha}\right)^{2}\left(3 L_{3}(1)-3 M_{2}(2)\right)+\left(\theta+\frac{\beta}{\alpha}\right)^{3} M_{3}(3) \\
+3 \theta\left(\theta+\frac{\beta}{\alpha}\right) L_{3}(1)+\theta\left(4 E_{\mu}\left(3 \alpha T^{\mu}\right)-3 E_{\mu}\left(2 \alpha T^{\mu}\right)\right)
\end{aligned}
$$

where $L_{3}(1)=E_{\mu}\left(3 \alpha T^{\mu}\right)-E_{\mu}\left(2 \alpha T^{\mu}\right) E_{\mu}\left(\alpha T^{\mu}\right)$ and $L_{3}(2)=E_{\mu}\left(\alpha T^{\mu}\right)^{2}-$ $E_{\mu}\left(2 \alpha T^{\mu}\right)$. Note here that $M_{3}(2)=3 L_{3}(1)-3 M_{2}(2)$. Namely, there appear the nonlinear modes of evolution $M_{3}(1), L_{3}(1), M_{2}(2)$ and $M_{3}(3)$. Among them, $L_{3}(1), M_{2}(2)$ and $M_{3}(3)$ tend to vanish as $\mu \rightarrow 1$. Also, the last term in Eq. $(55) \theta\left(4 E_{\mu}\left(3 \alpha T^{\mu}\right)-3 E_{\mu}\left(2 \alpha T^{\mu}\right)\right)$ is the new additional one.

\subsection{Characterization of anomalous diffusion}

Mathematical descriptions of anomalous diffusion have been discussed with the use of deterministic and stochastic equations in complex systems [20-22]. The coefficient of anomalous diffusion $\gamma$ is related to the scaling exponent $\ell$ of the detrended fluctuation analysis (DFA) [21] as $\gamma=\ell / 2$. In the case of the fractional Poisson process, the coefficient of anomalous diffusion $\mu$ can be estimated from the scaling exponent of the Fano factor as shown in Fig.7. On the other hand, one needs to identify the coefficient $\mu$ with the use of the nonlinear modes of evolution described by the Mittag-Leffler function in the limit of large $T$ in Figs 1, 2 and 4 due to the multi-fractal nature. 
From the expressions of the mean, the variance and the third order cumulants, one can understand the importance in considering the distribution of initial values for the generalized birth process, especially when the initial value is not controllable in real experiments. The mean value is characterized only by $M_{1}(1)$. It is difficult to identify the process only with the information of the mean evolution. On the other hand, one can distinguish the nature of the FGPP form the FYFP and the FPP based on the information of fluctuations (the higher order cumulants) with nonlinear evolution modes $M_{2}(1), M_{2}(2)$, $M_{3}(1), L_{3}(1)$ and $M_{3}(3)$.

\section{$5 \quad$ Summary and Remarks}

A fractional generalized pure birth process was studied based on the master master equation approach. The exact analytic solution for the process was given in terms of the Gauss hypergeometric function. The equations of moments and the cumulants were also obtained in the explicit form by the generating function. Further, the effect of distribution of the initial Poisson law was also elucidated. Numerical examples are given for displaying the features of the FGBP with the use of the Fano factor. The fractional generalization can tell us how the effect of memory influences the observed features of fluctuation. The appearance of composite fractional time evolutions of cumulants are displayed in comparison with the FPP which can give us a new method how to discriminate the FYFP from the fractional GBP.

The fractional generalized birth-death process is investigated from the composite fractional time evolutions, which will be published elsewhere. It is a challenging problem to perform fractional generalization, and to apply the method of theoretical analysis to multiple-component complex stochastic systems [23].

ACKNOWLEDGEMENTS. This work is partially supported by the JSPS, No. 24650147. One of the authors (I. P.) is also supported by JSPS, the Invitation Fellowship for Research in Japan No. S-13061.

\section{References}

[1] R. Metzler and J. Klafter, Phys. Rep., 339 (2000), 1-77.

[2] I. M. Sokolov, J. Klafter and A. Blumer, Phys. Today, November, (2002), 48-54. 
[3] J. L. Lebowitz and E. W. Montroll, On the wonderful world of random walks, in Nonequilibrium Phenomena II: From Stochastic to Hydrodynamics, E. W. Montroll and M. F. Schlesinger, Editors., Chapter 1, pp. 1-121, North-Holland, Amsterdam, The Netherlands, 1984.

[4] S. B. Lowen and M. C. Teich, Fractal-Based Point Processes, Wiley, New York, 2005.

[5] A. I. Saichev and G. M. Zaslavsky, Chaos, 7 (1997), 753-764.

[6] N. Laskin, Comm. Nonlin. Sci. and Numer. Sim., 8 (2003) ,201-213.

[7] E. Orsinger and F. Polito, Bernoulli, 16 (2010), 858-811.

[8] E. Orsinger and F. Polito, Bernoulli, 17 (2011), 114-137.

[9] H. Konno, Adv. Stud. Theor. Phys., 6 (2012), 1039-1058.

[10] E. Barkai, Phys. Rev., E63 (2001), 046118 1-17.

[11] T. Franosch, M. Grimm, M. Belushkin, F. Mor, G. Foffi, L. Forro and S. Jeney, Nature, 85 (2011), 85-88.

[12] H. Konno, Adv. Math. Phys., 2010 (2010), Article ID 504267, 1-12.

[13] H. Konno, Fractal fluctuations in the generalized Polya process, (2012), unpublised.

[14] R. Kubo, The fluctuation-dissipation theorem, Reports on Progress in Physics, 29 (1966), 255-284.

[15] H. Mori, Progr. Theor. Phys., 33 (1965), 423-455.

[16] R. Kubo, J. Math. Phys., 4 (1963), 174-183.

[17] M. Tokuyama and H. Mori, Prog. Theor. Phys., 55 (1976), 411-429.

[18] T. Bedford and R. Cooke, Probabilistic Risk Analysis, Cambridge Univ. Press, New York, 2001.

[19] W. Feller, An Introduction to Probability Theory and Its Applications, Wiley, New York, 1968.

[20] G. Espinosa-Paredes, M. A. Polo-Labarrios and J. Alvarez-Ramirez, Ann. Nucl. Energy, 54 (2013), 227-232.

[21] C.-K. Peng et al., Mosaic organization of DNA nucleotides, Phys. Rev., E49 (1994), 1685-1689. 
[22] F. Santamaria, et al., Anomalous Diffusion in Purkinje Cell Dendrites Caused by Spines, Neuron, 52 (2006), 635-648.

[23] I. Pázsit and L. Pál, Neutron Fluctuations, Elsevier, New York, 2008.

\section{Appendix A}

\section{Integral Kernel of Lévy Transform and Its Laplace Transform}

The density of the one-sided Lévy distribution $\ell_{\mu}(z)$ is expressed in terms of the Gamma function as

$$
\ell_{\mu}(z)=\frac{1}{2 \pi i} \int_{c-i \infty}^{c+i \infty} \frac{\Gamma(1 / \mu-s / \mu)}{\mu \Gamma(1-s)} z^{-s} d s
$$

In terms of Fourier series, (A1) is written in the form:

$$
\ell_{\mu}(z)=\frac{1}{\pi} \sum_{n=0}^{\infty} \frac{\Gamma(1+n \mu)}{n !}(-1)^{n} \sin (\pi n \mu) z^{-1+n \mu}
$$

The cumulative distribution should be integrated in the range $[0, t]$ as

$$
L_{\mu}(t)=\int_{0}^{t} \ell_{\mu}(z) d z
$$

Therefore, the kernel function in Eq.(9) is expressed as

$$
K_{\mu}(\tau, t)=\frac{d}{d \tau}\left[1-L_{\mu}\left(t / \tau^{1 / \mu}\right)\right]=\frac{1}{\mu} \frac{t}{\tau^{1+1 / \mu}} \ell_{\mu}\left(\frac{t}{\tau^{1 / \tau}}\right)
$$

The Laplace transform of the kernel function (A4) is given by

$$
\hat{K}_{\mu}[\tau, s]=\int_{0}^{\infty} e^{-s t} K_{\mu}(\tau, t) d t=s^{\mu-1} \exp \left(-s^{\mu} \tau\right)
$$

\section{Appendix B}

\section{Derivation of the solution of Eq.(12) with the Gauss hypergeometric function}

The Laplace transform of the generating function $g_{\mu}[z, s]$ with the initial condition $g_{\mu}(z, 0)=z^{n_{0}}$ is subject to

$$
z(z-1) \frac{d}{d z} g_{\mu}[z, s]+\frac{\beta}{\alpha}(z-1) g_{\mu}[z, s]=\frac{s^{\mu}}{\alpha} g_{\mu}[z, s]-\frac{s^{\mu-1}}{\alpha} z^{n_{0}} .
$$


Rewriting (B1) leads to the differential equation with an external force:

$$
z(1-z) \frac{d}{d z} g_{\mu}[z, s]+\left(\frac{s^{\mu}}{\alpha}+\frac{\beta}{\alpha}-\frac{\beta}{\alpha} z\right) g_{\mu}[z, s]-\frac{s^{\mu-1}}{\alpha} z^{n_{0}}=0 .
$$

Let us put $g_{\mu}[z, s]=z^{z_{0}} H_{\mu}[z, s]$ to eliminate the external force. Then, taking derivative with respect to $z$, we have

$$
\begin{aligned}
z(1-z) \frac{d^{2}}{d z^{2}} H_{\mu}[z, s]+ & {\left[n_{0}+1+\frac{s^{\mu}}{\alpha}+\frac{\beta}{\alpha}-\left(n_{0}+\frac{\beta}{\alpha}+2\right) z\right] \frac{d}{d z} H_{\mu}[z, s] } \\
& -\left(n_{0}+\frac{\beta}{\alpha}\right) H_{\mu}[z, s]=0 .
\end{aligned}
$$

This is the Gauss hypergeometric differential equation with the set of parameters

$$
(a, b, c)=\left(n_{0}+\frac{\beta}{\alpha}, 1, n_{0}+1+\frac{s^{\mu}}{\alpha}+\frac{\beta}{\alpha}\right) .
$$

The solution takes the form: $g_{\mu}[z, s]=g_{0} z^{z_{0}} F(a, b, c ; z)$, where $g_{0}$ is determined from the constraint $g_{\mu}[z=1, s]=1 / s$ as $g_{0}=s^{\mu-1} /\left(s^{\mu}+\alpha n_{0}+\beta\right)$. This solution is exactly identical with the one which is derived from Eq.(1) by the inverse Lévy transform.

\section{Appendix $\mathrm{C}$}

\section{Derivation of the expressions of moments in Eqs.(34)-(36)}

Taking derivative of $\overline{g_{\mu}[z, s]}$ with respect to $z$ once, we have

$$
\begin{gathered}
\overline{g_{\mu}^{\prime}[z, s]}=\frac{s^{\mu-1}}{\alpha}\left(\frac{\beta}{\alpha}\right) \int_{0}^{1} x(1-x)^{\frac{s^{\mu}}{\alpha}+\frac{\beta}{\alpha}-1}(1-x z)^{-\frac{\beta}{\alpha}-1} \exp \left(-\frac{1-z}{1-x z} \theta\right) d x \\
+\frac{s^{\mu-1}}{\alpha} \theta \int_{0}^{1}(1-x)^{\frac{s^{\mu}}{\alpha}+\frac{\beta}{\alpha}-1}(1-x z)^{-\frac{\beta}{\alpha}-1} \exp \left(-\frac{1-z}{1-x z} \theta\right) d x \\
-\frac{s^{\mu-1}}{\alpha} \theta(1-z) \int_{0}^{1} x(1-x)^{\frac{s^{\mu}}{\alpha}+\frac{\beta}{\alpha}-1}(1-x z)^{-\frac{\beta}{\alpha}-2} \exp \left(-\frac{1-z}{1-x z} \theta\right) d x .
\end{gathered}
$$

Substituting $z=1$ in Eq.(C1), one obtains

$$
\begin{gathered}
\overline{g^{\prime}[z=1, s]}=\left(\frac{s^{\mu-1}}{\alpha}\right)\left(\frac{\beta}{\alpha}\right) B\left(2, \frac{s^{\mu-1}}{\alpha}-1\right)+\left(\frac{s^{\mu-1}}{\alpha}\right) \theta B\left(1, \frac{s^{\mu}}{\alpha}-1\right) \\
=\left(\frac{\beta}{\alpha}\right)\left(\frac{s^{\mu-1}}{s^{\mu}-\alpha}-\frac{1}{s}\right)+\theta \frac{s^{\mu-1}}{s^{\mu}-\alpha} .
\end{gathered}
$$




$$
\overline{\langle n(t)\rangle}=\theta E_{\mu}\left(\alpha t^{\mu}\right)+\left(\frac{\beta}{\alpha}\right)\left(E_{\mu}\left(\alpha t^{\mu}\right)-1\right) .
$$

Similarly, taking derivative of $\overline{g_{\mu}[z, s]}$ with respect to $z$ twice, we have

$$
\begin{gathered}
\overline{g^{\prime \prime}[z, s]}=\frac{s^{\mu-1}}{\alpha}\left(\frac{\beta}{\alpha}\right)^{2} \int_{0}^{1} x^{2}(1-x)^{\frac{s^{\mu}}{\alpha}+\frac{\beta}{\alpha}-1}(1-x z)^{-\frac{\beta}{\alpha}-2} \exp \left(-\frac{1-z}{1-x z} \theta\right) d x \\
+\frac{s^{\mu-1}}{\alpha}\left(\frac{\beta}{\alpha}\right) \int_{0}^{1} x^{2}(1-x)^{\frac{s^{\mu}}{\alpha}+\frac{\beta}{\alpha}-1}(1-x z)^{-\frac{\beta}{\alpha}-2} \exp \left(-\frac{1-z}{1-x z} \theta\right) d x \\
+\frac{s^{\mu-1}}{\alpha} 2\left(\frac{\beta}{\alpha}\right) \theta \int_{0}^{1} x(1-x)^{\frac{s^{\mu}}{\alpha}+\frac{\beta}{\alpha}-1}(1-x z)^{-\frac{\beta}{\alpha}-2} \exp \left(-\frac{1-z}{1-x z} \theta\right) d x \\
-\frac{s^{\mu-1}}{\alpha} 2\left(\frac{\beta}{\alpha}\right) \theta(1-z) \int_{0}^{1} x^{2}(1-x)^{\frac{s^{\mu}}{\alpha}+\frac{\beta}{\alpha}-1}(1-x z)^{-\frac{\beta}{\alpha}-3} \exp \left(-\frac{1-z}{1-x z} \theta\right) d x \\
+\frac{s^{\mu-1}}{\alpha} \theta^{2}(1-z)^{2} \int_{0}^{1} x^{2}(1-x)^{\frac{s^{\mu}}{\alpha}+\frac{\beta}{\alpha}-1}(1-x z)^{-\frac{\beta}{\alpha}-4} \exp \left(-\frac{1-z}{1-x z} \theta\right) d x . \\
-\frac{s^{\mu-1}}{\alpha} 2 \theta \theta_{0}^{1} x(1-x) \int_{0}^{1} x(1-x)^{\frac{s^{\mu}}{\alpha}+\frac{\beta}{\alpha}-1}(1-x z)^{-\frac{\beta}{\alpha}-3} \exp \left(-\frac{s^{\mu}}{1-\frac{\beta}{\alpha}-1}(1-x z)^{-\frac{\beta}{\alpha}-2} \exp \left(-\frac{1-z}{1-x z} \theta\right) d x\right. \\
+\frac{s^{\mu-1}}{\alpha} \theta^{2} \int_{0}^{1}(1-x)^{\frac{s^{\mu}}{\alpha}+\frac{\beta}{\alpha}-1}(1-x z)^{-\frac{\beta}{\alpha}-2} \exp \left(-\frac{1-z}{1-x z} \theta\right) d x \\
-\frac{s^{\mu-1}}{\alpha} 2 \theta(1-z) \int_{0}^{1} x^{2}(1-x)^{\frac{s^{\mu}}{\alpha}+\frac{\beta}{\alpha}-1}(1-x z)^{-\frac{\beta}{\alpha}-3} \exp \left(-\frac{1-z}{1-x z} \theta\right) d x
\end{gathered}
$$

Substituting $z=1$ in Eq.(C4), one obtains

$$
\overline{g^{\prime \prime}[z=1, s]}=\left(\frac{s^{\mu-1}}{\alpha}\right)\left(\frac{\beta}{\alpha}\right)^{2} B\left(3, \frac{s^{\mu}}{\alpha}-2\right)+\left(\frac{s^{\mu-1}}{\alpha}\right)\left(\frac{\beta}{\alpha}\right) B\left(3, \frac{s^{\mu}}{\alpha}-2\right)
$$




$$
\begin{aligned}
& +\left(\frac{s^{\mu-1}}{\alpha}\right) 2 \theta\left(\frac{\beta}{\alpha}\right) B\left(2, \frac{s^{\mu}}{\alpha}-2\right)+\left(\frac{s^{\mu-1}}{\alpha}\right) 2 \theta B\left(2, \frac{s^{\mu}}{\alpha}-2\right)+\left(\frac{s^{\mu-1}}{\alpha}\right) \theta^{2} B\left(1, \frac{s^{\mu}}{\alpha}-2\right) \\
& \overline{\left\langle n(t)^{2}\right\rangle}=\left(\left(\theta+\frac{\beta}{\alpha}\right)^{2}+2 \theta+\frac{\beta}{\alpha}\right) E_{\mu}\left(2 \alpha t^{\mu}\right)-\left(\theta+\frac{\beta}{\alpha}\right)\left(1+2 \frac{\beta}{\alpha}\right) E_{\mu}\left(\alpha t^{\mu}\right)+\left(\frac{\beta}{\alpha}\right)^{2} .
\end{aligned}
$$

In deriving these results, the mathematical formulae for the Beta function are utilized: $B\left(3, \frac{s^{\mu}}{\alpha}-2\right)=\frac{2 \alpha^{3}}{s^{\mu}\left(s^{\mu}-\alpha\right)\left(s^{\mu}-2 \alpha\right)}, B\left(2, \frac{s^{\mu}}{\alpha}-2\right)=\frac{\alpha^{2}}{\left(s^{\mu}-\alpha\right)\left(s^{\mu}-2 \alpha\right)}$ and $B\left(1, \frac{s^{\mu}}{\alpha}-2\right)=\frac{\alpha}{\left(s^{\mu}-2 \alpha\right)}$. It can be shown that the expression of the variance reduces to

$$
\begin{gathered}
\overline{\sigma_{n}^{2}(T)}=\left(\theta+\frac{\beta}{\alpha}\right)\left(E_{\mu}\left(2 \alpha T^{\mu}\right)-E_{\mu}\left(\alpha T^{\mu}\right)\right)+\left(\theta+\frac{\beta}{\alpha}\right)^{2}\left(E_{\mu}\left(2 \alpha T^{\mu}\right)-E_{\mu}\left(\alpha T^{\mu}\right)^{2}\right) \\
+\theta E_{\mu}\left(2 \alpha T^{\nu}\right) .
\end{gathered}
$$

The last term in Eq.(C6) is the additional term which can be ascribed to the averaging effect over the Poisson distribution of the initial value with the parameter $\theta$.

\section{Received: December 11, 2013}

